\title{
A bi-objective inspection policy optimization model for finite-life repairable systems using a genetic algorithm
}

\author{
Ramadan, S. $^{\mathrm{a},{ }^{*}}$ \\ ${ }^{a}$ Department of Mechanical and Industrial Engineering, Applied Science Private University, Shafa Badran, Amman, Jordan
}

\begin{abstract}
A B S T R A C T
This paper presents a bi-objective optimization model for finding the optimal number and optimal aperiodic times for the inspections of finite-life repairable systems when the availability of the component and the total maintenance cost are under consideration. The model utilizes the delay-time concept under perfect inspection assumption. The defect arrival process is modelled using the nonhomogeneous Poisson process and the failure times are probabilistic. The solution to this problem is NP-hard, therefore, a mutation-based genetic algorithm has been designed to solve the model. The effectiveness of the model was demonstrated using seven illustrative examples and compared to an existing classical periodic inspection model that uses a fixed number of inspections. The results showed that the proposed model did better (in all of the attributes) than the aperiodic model that using a fixed number of inspections. Furthermore, the results showed that the proposed model gave better results than a single-objective aperiodic model. The proposed model is a general model that can be implemented with different rates of occurrence of defects and different delay-time distributions. Also this model can be extended easily to cover complex systems and imperfect inspection cases.
\end{abstract}

\section{ARTICLE INFO}

Keywords:

Maintenance

Aperiodic inspection

Periodic inspection

Delay-time

Multi-objective optimization

Genetic algorithms

*Corresponding author:

s_ramadan@asu.edu.jo

(Ramadan, S.)

Article history:

Received 14 September 2015

Revised 29 September 2015

Accepted 19 October 2015 


\section{References}

[1] Dhillon, B.S. (2002). Engineering maintenance: A modern approach, CRC press, Boca Raton, USA, doi: 10.1201/ 9781420031843.

[2] Moubray J. (1997). Reliability-centered Maintenance II, (2nd edition), Industrial Press, New York, USA.

[3] Barlow, E.E., Proschan, F., Hunter, L.C. (1965). Mathematical theory of reliability, Wiley, New York-LondonSydney, doi: 10.1002/bimj.19660080409.

[4] Wild, R. (1985). Essentials of production and operations management, Holt, Rinehart \& Winston, London, UK.

[5] Dhillon, B.S. (1988). Mechanical reliability: Theory, models, and applications, American Institute of Aeronautics and Astronautics, Washington, D.C., USA.

[6] Jardine, A.K.S. (1973). Maintenance, replacement and reliability, Pitman Publishing, London, UK.

[7] Dhillon, B.S. (1983). Systems reliability, maintainability, and management, Petrocelli Books, New York, USA.

[8] Christer, A.H. (1976). Innovative decision making, In: Proceedings of the NATO Conference on the Role and Effectiveness of Theories of Decision in Practice, Hodder \& Stoughton, 368-377.

[9] Christer, A.H., Waller, W.M. (1984). Delay time models of industrial inspection maintenance problems, The Journal of the Operational Research Society, Vol. 35, 401-406, doi: 10.1057/jors.1984.80.

[10] Christer, A.H. (1987). Delay-time model of reliability of equipment subject to inspection monitoring, The Journal of the Operational research Society, Vol. 38, No. 4, 329-334, doi: 10.2307/2582056.

[11] Baker, R.D., Christer, A.H. (1994). Review of delay-time OR modelling of engineering aspects of maintenance, European Journal of Operational Research, Vol. 73, No. 3, 407-422, doi: 10.1016/0377-2217(94)90234-8.

[12] Christer, A.H. (1999). Developments in delay time analysis for modelling plant maintenance, Journal of Operational Research Society, Vol. 50, 1120-1137, doi: 10.1057/palgrave.jors.2600837.

[13] Christer, A.H., Wang, W., Baker, R.D., Sharp, J. (1995). Modelling maintenance practice of production plant using the delay-time concept, IMA Journal of Management Mathematics, Vol. 6, No. 1, 67-84, doi: 10.1093/imaman/ 6.1.67.

[14] Wang, W., Majid, H.B.A. (2000). Reliability data analysis and modelling of offshore oil platform plant, Journal of Quality in Maintenance Engineering, Vol. 6, No. 4, 287-295, doi: 10.1108/13552510010346824.

[15] Dawotola, A.W., Trafalis, T.B., Mustaffa, Z., van Gelder, P.H.A.J.M., Vrijling, J.K. (2013). Risk-based maintenance of a cross-country petroleum pipeline system, Journal of Pipeline Systems Engineering and Practice, Vol. 4, No. 3, 141-148, doi: 10.1061/(ASCE)PS.1949-1204.0000121.

[16] Abdel-Hameed, M. (1995 ). Correction to: 'Inspection and maintenance policies of devices subject to deterioration', Advances in Applied Probability, Vol. 27, No. 2, p. 584.

[17] Okumura, S., Jardine, A.K.S., Yamashina, H. (1996). An inspection policy for a deteriorating single-unit system characterized by a delay-time model, International Journal of Production Research, Vol. 34, No. 9, 2441-2460, doi: $10.1080 / 00207549608905037$.

[18] Okumura, (1997). An inspection policy for deteriorating processes using delay-time concept, International Transactions in Operational Research, Vol. 4, No. 5-6, 365-375.

[19] Wang, W. (2008). Delay time modelling, In: Kobbacy, K.A.H., Murthy, D.N.P. (eds.), Complex system maintenance handbook, Springer Series in Reliability Engineering, Springer, London, 345-370, doi: 10.1007/978-1-84800011-7 14.

[20] Wang, W. (2009). An inspection model for a process with two types of inspections and repairs, Reliability Engineering \& System Safety, Vol. 94, No. 2, 526-533, doi. 10.1016/i.ress.2008.06.010.

[21] Wang, W., Banjevic, D., Pecht, M. (2010). A multi-component and multi-failure mode inspection model based on the delay time concept, Reliability Engineering and System Safety, Vol. 95, No. 8, 912-920, doi: 10.1016/j.ress. 2010.04.004.

[22] Taghipour, S., Banjevic, D., Jardine, A.K.S. (2010). Periodic inspection optimization model for a complex repairable system, Reliability Engineering \& System Safety, Vol. 95, No. 9, 944-952, doi: 10.1016/j.ress.2010.04.003.

[23] Taghipour, S., Banjevic, D. (2011). Periodic inspection optimization models for a repairable system subject to hidden failures, IEEE Transactions on Reliability, Vol. 60, No. 1, 275-285, doi: 10.1109/TR.2010.2103596.

[24] Taghipourab, S., Banjevic, D. (2012). Optimum inspection interval for a system under periodic and opportunistic inspections, IIE Transactions, Vol. 44, No. 11, 932-948, doi: 10.1080/0740817X.2011.618176.

[25] Kakade, V., Valenzuela, J.F., Smith, J.S. (2004). An optimization model for selective inspection in serial manufacturing systems, International Journal of Production Research, Vol. 42, No. 18, 3891-3909, doi: $10.1080 / 00207540410001704014$.

[26] Golmakani, H.R., Moakedi, H. (2012). Periodic inspection optimization model for a multi-component repairable system with failure interaction, The International Journal of Advanced Manufacturing Technology, Vol. 61, No. 1, 295-302, doi: 10.1007/s00170-011-3693-8.

[27] Wang, W., Christer, A.H. (2003). Solution algorithms for a nonhomogeneous multi-component inspection model, Computers \& Operations Research, Vol. 30, No. 1, 19-34, doi: 10.1016/S0305-0548(01)00074-0.

[28] Chan, G.K., Asgarpoor, S. (2006). Optimum maintenance policy with Markov processes, Electric Power Systems Research, Vol. 76, No. 6-7, 452-456, doi: 10.1016/j.epsr.2005.09.010.

[29] Von Alven, W.H. (1964). Reliability Engineering, Prentice-Hall, Englewood Cliffs, New Jersey, USA.

[30] Nazemi, E., Shahanaghi, K. (2015). Developing an inspection optimization model based on the delay-time concept, Journal of Industrial Engineering, Vol. 2015, Article ID 843137, 7 pages, doi: 10.1155/2015/843137.

[31] Ben-Daya, M., Duffuaa, S.O., Raouf, A., Knezevic, J., Ait-Kadi, D. (2009). Handbook of maintenance management and engineering, Springer, London, UK, doi: 10.1007/978-1-84882-472-0. 
[32] Christer, A.H., Wang, W. (1995). A delay-time-based maintenance model of a multi-component system, IMA Journal of Management Mathematics, Vol. 6, No. 2, 205-222, doi: 10.1093/imaman/6.2.205.

[33] Wang, W., Majid, H.B.A. (2000). Reliability data analysis and modelling of offshore oil platform plant, Journal of Quality in Maintenance Engineering, Vol. 6, No. 4, 287-295, doi: 10.1108/13552510010346824. 


\title{
Dvokriterijski model nadzorne strategije za optimizacijo vzdrževanja popravljivih izdelkov z uporabo genetskega algoritma
}

\author{
Ramadan, S. ${ }^{a,{ }^{*}}$ \\ ${ }^{a}$ Department of Mechanical and Industrial Engineering, Applied Science Private University, Shafa Badran, Amman, Jordan
}

\section{POVZETEK}

$\mathrm{V}$ članku je predstavljen dvokriterijski optimizacijski model za iskanje optimalnega števila in časa neperiodičnih pregledov za inšpekcijo popravljivih sistemov oziroma izdelkov. V ozir so vzeti razpoložljivost sistema oziroma komponente ter skupni stroški vzdrževanja. Model izkorišča koncept časa zastoja ob idealnih inšpekcijskih predpostavkah. Pojavljanje okvar je modelirano z uporabo nehomogenega Poissonovega procesa, časi nedelovanja pa se ravnajo po verjetnostnih načelih. Ker gre za t.i. NP-težki problem, je bil zasnovan genetski algoritem, ki temelji na operaciji mutacije. Učinkovitost modela je predstavljena s pomočjo sedmih zgledov, narejena pa je tudi primerjava z obstoječim klasičnim modelom periodičnih pregledov, ki vključuje konstantno število pregledov. Rezultati so pokazali, da je predlagan model (po vseh lastnostih) boljši kot aperiodični model, ki vključuje konstantno število pregledov, poleg tega pa predlagan model daje boljše rezultate kot enokriterijski aperiodični model. Predlagan model je splošen in se lahko uporabi z različnimi stopnjami pojavljanja napak in različnimi porazdelitvami časov zastojev. Model se lahko na preprost način razširi, da omogoča reševanje problemov pri kompleksnih sistemih in pri neidealnih inšpekcijskih primerih.
\end{abstract}

PODATKI O ČLANKU

Ključne besede:

Vzdrževanje

Neperiodično spremljanje

Periodično spremljanje

Čas zastoja

Večkriterijska optimizacija

Genetski algoritmi

*Kontaktna oseba:

s_ramadan@asu.edu.jo

(Ramadan, S.)

Zgodovina članka:

Prejet 14. septembra 2015

Popravljen 29. septembra 2015

Sprejet 19. oktobra 2015 